\title{
THE
}

1971

\section{Ising Chain with Competing Interactions in a Staggered Field}

John F. Nagle

Jill C. Bonner

University of Rhode Island

Follow this and additional works at: https://digitalcommons.uri.edu/phys_facpubs

Terms of Use

All rights reserved under copyright.

\section{Citation/Publisher Attribution}

Nagle, J. F., \& Bonner, J. C. (1971). Ising Chain with Competing Interactions in a Staggered Field. J. Chem. Phys. 54(2), 729. doi: 10.1063/1.1674903

Available at: http://dx.doi.org/10.1063/1.1674903

This Article is brought to you for free and open access by the Physics at DigitalCommons@URI. It has been accepted for inclusion in Physics Faculty Publications by an authorized administrator of DigitalCommons@URI. For more information, please contact digitalcommons-group@uri.edu. 


\title{
A|P| $\begin{aligned} & \text { The Journal of } \\ & \text { Chemical Physics }\end{aligned}$
}

\section{Ising Chain with Competing Interactions in a Staggered Field}

\author{
J. F. Nagle and J. C. Bonner
}

Citation: The Journal of Chemical Physics 54, 729 (1971); doi: 10.1063/1.1674903

View online: http://dx.doi.org/10.1063/1.1674903

View Table of Contents: http://scitation.aip.org/content/aip/journal/jcp/54/2?ver=pdfcov

Published by the AIP Publishing

\section{Articles you may be interested in}

Modulated Phase of an Ising System with Competing Interactions on a Cayley Tree of Arbitrary Order AIP Conf. Proc. 1281, 2074 (2010); 10.1063/1.3498359

Ordering in two-dimensional Ising models with competing interactions

Low Temp. Phys. 31, 722 (2005); 10.1063/1.2008132

An Ising model with three competing interactions on a Cayley tree

J. Math. Phys. 45, 3645 (2004); 10.1063/1.1781747

Solitons and phasons in the Ising model with competing interactions

J. Appl. Phys. 50, 7409 (1979); 10.1063/1.326910

Cubic spin model with competing bilinear and higher-degree pair interactions on a linear chain in a magnetic field

J. Appl. Phys. 50, 2037 (1979); 10.1063/1.327100

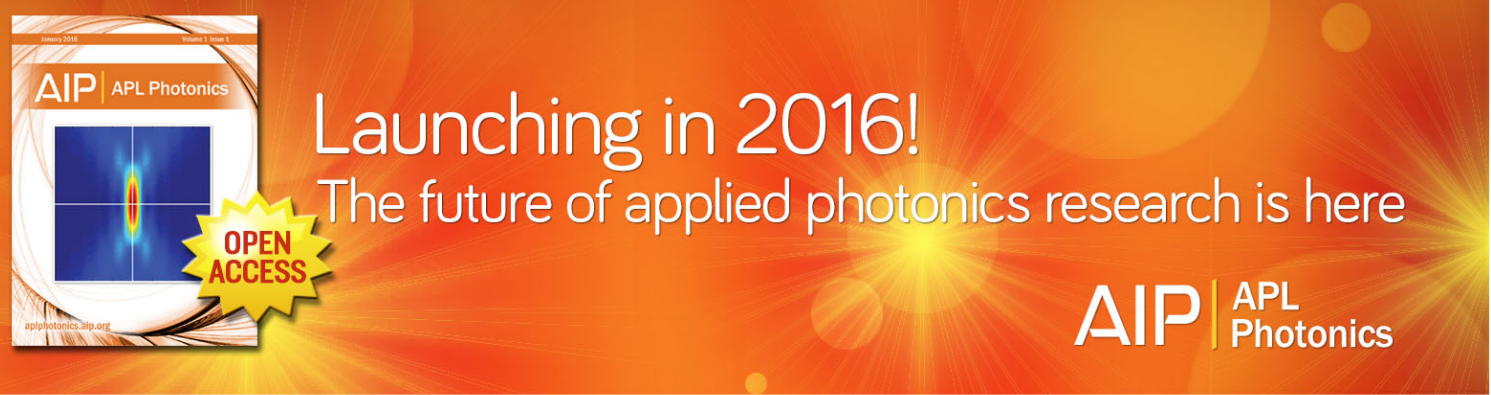




\title{
Ising Chain with Competing Interactions in a Staggered Field*
}

\author{
J. F. Nagle $\dagger$ and J. C. Bonner \\ Depariment of Physics, Carnegie-Mellon University, Pittsburgh, Pennsylvania 15213
}

(Received 28 July 1970)

\begin{abstract}
Work on the Ising chain with competing interactions is extended to the case where a staggered field is included as well as the usual direct field. A simple transformation enables one to interpret the behavior of this model as a metamagnet. Tricritical points are found and analyzed, and the observability of the tricritical exponents, as distinguished from the usual critical exponents, is discussed.
\end{abstract}

\section{INTRODUCTION}

In a previous paper ${ }^{1}$ (to be called $I$ in this paper) the one-dimensional spin $-1 / 2$ Ising chain with competing nearest neighbor and very long range equivalent neighbor interactions was discussed. The Hamiltonian may be written

$\mathfrak{H C}=-J_{\mathrm{SR}} \sum_{i=1}^{N}\left(\mu_{i} \mu_{i+1}\right)-\frac{J_{\mathrm{LR}}}{N} \sum_{i=1}^{N} \sum_{j=1}^{N} \mu_{i} \mu_{j}-H \sum_{i=1}^{N} \mu_{i}$,

where $\mu_{i}= \pm 1$. For the case of competing interactions, $J_{\mathrm{SR}}<0$ and $J_{\mathrm{LR}}>0$. One reason for choosing the somewhat artificial very long range equivalent neighbor interaction is, of course, that the complete thermodynamic solution to such a Hamiltonian can be found relatively easily provided that the $J_{\mathrm{LR}}=0$ problem can be solved in a field. ${ }^{2-5}$ The $J_{\mathrm{LR}}=0$ problem can be solved quite easily for the one-dimensional Ising model with short range interactions and also for some one-dimensional quantum mechanical models ${ }^{6}$ as well as for one-dimensional continuum systems. ${ }^{7}$ Further, the equivalent neighbor interaction guarantees the existence of a phase transition. Also, the equivalent neighbor and nearest neighbor interactions are opposite extremes in terms of the range of interactions.

One interesting feature of the solution of the Hamiltonian in Eq. (1) is the existence of a tricritical point, which is defined to be the point at which three critical lines meet. ${ }^{8}$ (A critical line is a line of critical points.) The confluent point, found and defined in (I), appears as a tricritical point in a phase diagram in which $H, T$, and $J_{\mathrm{LR}}$ are variables. However, it can be argued that $J_{\mathrm{LR}}$ represents a spin interaction strength and thus is not a thermodynamic variable. In this paper another term is added to the Hamiltonian, namely,

$$
-H^{\prime} \sum_{i=1}^{N}(-1)^{i} \mu_{i}
$$

This term represents the effect of a staggered field which operates in one sense on one sublattice consisting of even-numbered spins and in the opposite sense on the other sublattice consisting of the odd-numbered spins. (Although such a field is most easily realized theoretically, it is at least a more realistic variable than $J_{\mathrm{LR}}$.) In this paper we discuss the tricritical points in the $H, H^{\prime}, T$ phase diagram. It is interesting that in these diagrams the confluent point found in I becomes a tetracritical point. It might be noted that the addition of a staggered field does not present any real difficulty in solving the problem, as was shown by Stout and Chisholm. ${ }^{3}$

The introduction of the staggered field also facilitates interpreting the results of this work in terms of the properties of metamagnets. ${ }^{3,9,10}$ After performing a transformation which flips alternate spins, the tricritical point on the $H, H^{\prime}, T$ phase diagram becomes the point at which the metamagnetic phase transition changes from first order to second order. Defining $T^{*}$ to be the tricritical temperature and $T_{N}$ to be the Neel temperature, the variation with $J_{\mathbf{S R}} / J_{\mathbf{L R}}$ of the ratio $T^{*} / T_{N}$ is discussed in Sec. III.

Although it is to be expected that models with very long range equivalent neighbor interactions will not quantitatively fit experiments, especially very near critical points, it is hoped that such models will provide a qualitative picture of significant features of real systems. One such feature is the qualitative variation of $T^{*} / T_{N}$ with $J_{\mathrm{SR}} / J_{\mathrm{LR}}$. Another is that the critical exponents ${ }^{11}$ for such models at the tricritical point are different from the exponents at usual critical points. In connection with this latter observation it is of some experimental interest to obtain an estimate of the size of the "tricritical region" in which such tricritical indices are observable. This is done in Sec. III, using our calculations of the sublattice magnetization and specific heat as a guide.

In the next section we summarize the details of obtaining the solution. The results of physical interest are presented in Sec. III.

\section{DETAILS OF THE SOLUTION}

We first solve the nearest-neighbor Ising chain problem in a staggered field, i.e., $J_{\mathrm{LR}}=0$ in Eqs. (1) and (2). One may use the usual transfer matrix technique except that the presence of the staggered field requires a different transfer matrix for even-odd (eo) transfers $(2 i \rightarrow 2 i+1)$ than for odd-even transfers $(2 i-1 \rightarrow 2 i)$, as was shown by Stout and Chisholm. ${ }^{3}$ Defining $K=J_{\mathrm{SR}} / k T, K_{\mathrm{LR}}=J_{\mathrm{LR}} / k T, L_{0}=H / k T$, and 
$L_{0}^{\prime}=H^{\prime} / k T$, we have for the transfer matrices

$$
V_{\mathrm{e} 0}=\left[\begin{array}{ll}
\exp \left(K+L_{0}\right) & \exp \left(-K-L_{0}{ }^{\prime}\right) \\
\exp \left(-K+L_{0}{ }^{\prime}\right) & \exp \left(K-L_{0}\right)
\end{array}\right]
$$

and

$$
V_{\text {oe }}=\left[\begin{array}{ll}
\exp \left(K+L_{0}\right) & \exp \left(-K+L_{0}^{\prime}\right) \\
\exp \left(-K-L_{0}^{\prime}\right) & \exp \left(K-L_{0}\right)
\end{array}\right] .
$$

The partition function per spin $Z$ is given by the square root of the largest eigenvalue of $V_{\mathrm{e} o} \times V_{\mathrm{oe}}$, i.e.,

$$
\begin{aligned}
& Z^{2}=e^{2 K} \cosh 2 L_{0}+e^{-2 K} \cosh 2 L_{0}{ }^{\prime} \\
& +\left[e^{4 K} \cosh 2 L_{0}+e^{-4 K} \cosh ^{2} 2 L_{0}{ }^{\prime}\right. \\
& \left.+2 \cosh 2 L_{0} \cosh 2 L_{0}^{\prime}+2(1-\cosh 4 K)\right]^{1 / 2}
\end{aligned}
$$

For convenience let us abbreviate the square root in Eq. (4) as sqrt. Then the magnetization per spin can be found to be

$$
M=\partial \ln Z / \partial L_{0}=e^{2 K} \sinh 2 L_{0} / \mathrm{sqrt}
$$

and the "staggered" or sublattice-magnetization per spin is

$$
M^{\prime}=\partial \ln Z / \partial L_{0}{ }^{\prime}=e^{-2 K} \sinh 2 L_{0}{ }^{\prime} / \text { sqrt. }
$$

Next, we discuss the formal changes in the solution when an equivalent neighbor term is added, i.e., $J_{\text {LR }} \neq 0$ in Eqs. (1) and (2). The formal changes are well known in the context of mean field theory (see, for example, Stout and Chisholm ${ }^{3}$ ) and have been established rigorously by Baker ${ }^{4}$ and Suzuki. ${ }^{5}$ In order to establish notation we give a quick derivation. Writing for the $J_{\mathrm{LR}}=0$, i.e., the nearest-neighbor case,

$$
Z_{\mathrm{nn}}=\sum_{\text {states }} \exp \left[-\beta\left(E_{s}-M_{s} H_{0}-M_{s}^{\prime} H_{0}^{\prime}\right)\right]
$$

and using a maximum term argument, we can assert that in the thermodynamic limit the free energy per spin is

$$
\begin{aligned}
& F_{\mathrm{nn}}\left(H_{0}, H_{0}{ }^{\prime}, T\right)=\min _{M, M^{\prime}}\left[E\left(M, M^{\prime}\right)\right. \\
& \left.-(k T / N) \operatorname{In} W\left(M, M^{\prime}\right)-M H_{0}-M^{\prime} H_{0}{ }^{\prime}\right],
\end{aligned}
$$

where $E\left(M, M^{\prime}\right)$ is an average energy per spin over the number $W\left(M, M^{\prime}\right)$ of states with magnetizations $M$ and $M^{\prime}$ given by (5) and (6). Exactly the same sort of thing can be done when $J_{\mathrm{LR}} \neq 0$ to give

$$
\begin{aligned}
& F_{\mathrm{LR}}\left(H, H^{\prime}, T\right)=\min _{M, M^{\prime}}\left[E\left(M, M^{\prime}\right)\right. \\
& \left.\quad-(k T / N) \ln W\left(M, M^{\prime}\right)-M H-M^{\prime} H^{\prime}-J_{\mathrm{LR}} M^{2}\right]
\end{aligned}
$$

and $E\left(M, M^{\prime}\right)$ and $W\left(M, M^{\prime}\right)$ are the same as in Eq. (8). Thus,

$$
\begin{aligned}
& F_{\mathrm{LR}}\left(H, H^{\prime}, T\right)=\min _{M, M^{\prime}}\left[F_{\mathrm{nn}}\left(H_{0}, H_{0}{ }^{\prime}, T\right)\right. \\
& \left.+M\left(H_{0}-H\right)+M^{\prime}\left(H_{0}{ }^{\prime}-H\right)-J_{\mathrm{LR}} M^{2} N\right],
\end{aligned}
$$

where now the variables $H_{0}$ and $H_{0}{ }^{\prime}$ are related to $M, M^{\prime}$, and $T$ via Eqs. (5) and (6).

Let us perform a double Legendre transformation:

$$
A\left(M, M^{\prime}, T\right)=F_{\mathrm{LR}}\left(H, H^{\prime}, T\right)+M H+M^{\prime} H^{\prime} .
$$

Then from Eqs. (11), (10), (6), (5), and

$$
H=(\partial A / \partial M)_{M^{\prime}, r^{\prime}} \text { and } H^{\prime}=\left(\partial A / \partial M^{\prime}\right)_{M, T},
$$

we have

$$
H=H_{0}-2 J_{\mathrm{LR}} M \quad \text { and } \quad H^{\prime}=H_{0} .
$$

Thus, given $H, H^{\prime}$, and $T$, the solution to the problem of finding the thermodynamic value of $M^{\prime}$ is given by Eq. (6), but the solution to the problem of finding $M$ requires minimizing $F_{\mathrm{LR}}$ in Eq. (10) as a function of $M$ under the constraint in Eq. (13). Instead, we prefer to define a mixed thermodynamic potential,

$$
G_{1}\left(M, H^{\prime}, T\right)=F_{\mathrm{LR}}\left(H, H^{\prime}, T\right)+M H,
$$

so that

$$
H=\left(\partial G_{1} / \partial M\right)_{\mathrm{H}^{\prime}, T} .
$$

It is then easily shown that the system is stable in a homogeneous phase with magnetization $M$ provided that the tangent to $G_{1}$ at $M$ lies completely below the $G_{1}$ versus $M$ curve; otherwise the system is metastable and will break up into two phases. This is the familiar double tangent or convex envelope prescription for obtaining thermodynamic behavior in the two phase region. ${ }^{12}$

It is not very useful to attempt analytic solutions of the regions of stability of the first-order phase transitions. It is far easier to carry out these computations numerically. The procedure was to compute $G_{1}\left(M, H^{\prime}, T\right)$ for a given $H^{\prime}$ and $T$ at some 40 values of $M$ (i.e., $H_{0}$ ). It was then tested whether or not the tangent to the $G_{1}$ curve at $M$ lies below the $G_{1}$ vs $M$ curve at the other computed values of $M$. This procedure provides an upper bound for the regions of stability. If the system was not stable for some values of $M$, an iteration scheme computed the boundary value of $M$ between the stable and metastable region to as many significant figures as desired. However, this numerical procedure breaks down near critical points because the unstable region may be so small as to be lost between two adjacent values of the original grid in $M$, but this is not important since the critical points can often be located analytically.

In order to locate critical points analytically it is most convenient to refer to the Landau free energy expansion, which is applicable because this is a classical model. For fixed $H^{\prime}$ this may be written,

$G_{1}\left(M, H^{\prime}, T\right)=a_{0}(T)+a_{2}(T)(\Delta M)^{2}$

and

$$
+a_{4}(T)(\Delta M)^{4}+a_{6}(T)(\Delta M)^{6}+\cdots
$$

$$
a_{n}(T)=\sum_{m=0}^{\infty} a_{n m}(\Delta T)^{m}
$$


This leads to an inverse differential susceptibility

$$
\begin{aligned}
1 / \chi=(\partial H / \partial M)_{T, \mathrm{H}^{\prime}}=2 a_{2}(T) & +12 a_{4}(T)(\Delta M)^{2} \\
& +30 a_{6}(T)(\Delta M)^{4}+\cdots .
\end{aligned}
$$

For $\Delta M=M-M_{c}$ this gives an infinite susceptibility when $a_{2}(T)=0$ and this feature locates the spinodal curves. As was discussed in Paper I the existence of a critical point in a field $H\left(H^{\prime}=0\right)$ requires that two spinodal curves meet. This results when two of the roots of the cubic equation $a_{2}(T)=0$ are equal. In the full $H \neq 0$ and $H^{\prime} \neq 0$ case the $a_{2}(T)=0$ equation becomes sixth-order and is not amenable to analytic expressions. However, in the present paper, we are most interested in the case $H=0$ and $H^{\prime} \neq 0$. This case reduces to a simple quadratic equation as follows:

$$
\left(1+\sinh ^{2} L_{0}{ }^{\prime}\right)\left(e^{4 K}+\sinh ^{2} L_{0}^{\prime}\right)=4 K_{\mathrm{LR}^{2} e^{8 K} .}
$$

But Eq. (17) only locates the critical point when the point $M=0, H^{\prime}, T$ is stable. In practice this is the case when $a_{4}(T)>0$ and is not the case when $a_{4}(T)<0$. The point $M=0, H^{\prime}$, and $T$ at which $a_{4}(T)=0$ is the tricritical point and has been found by simultaneous numerical solution of $a_{2}(T)=0$ in the form of Eq. (17) and $a_{4}(T)=0$. This procedure was verified for a test case, i.e., one particular $J_{\mathrm{LR}}$ and $J_{\mathrm{SR}}$, by detailed computation using $G_{1}\left(M, H^{\prime}, T\right)$ and the double tangent construction.

\section{THERMODYNAMIC BEHAVIOR}

First, let us relate this model to metamagnets. The interaction which is believed to induce the phase transition in metamagnets is antiferromagnetic. In the mean field approximation, i.e., in terms of the equivalent neighbor model this interaction appears $\mathrm{as}^{9}$

$$
\mathfrak{H}_{A}=N\left[-\Gamma\left(M_{1}^{2}+M_{2}^{2}\right)+2 A M_{1} M_{2}\right]
$$

where $M_{j}=\left(\sum \mu_{i}\right) / N$ over spins on the $j=1$ or 2 sublattice. We are interested in this paper in the special case $\Gamma=A=J_{\mathrm{LR}^{\prime}}$ for which

$$
\mathfrak{H}_{A}=-J_{\mathrm{LR}^{\prime}} N\left(M_{1}-M_{2}\right)^{2} \text {. }
$$

The whole Hamiltonian may then be written

$$
\begin{aligned}
\mathcal{H}=-J_{\mathrm{LR}^{\prime}} N & \left(M_{1}-M_{2}\right)^{2}-J_{\mathrm{SR}^{\prime}} \sum_{i} \mu_{i} \mu_{i+1} \\
& -N H\left(M_{1}+M_{2}\right)-N H^{\prime}\left(M_{1}-M_{2}\right) .
\end{aligned}
$$

If one now formally redefines all the spins on sublattice 2 to be their negatives, i.e., $\mu_{i}{ }^{(2)} \rightarrow-\mu_{i}{ }^{(2)}$, then we recover the Hamiltonian in Eqs. (1) and (2) provided that $J_{\mathrm{LR}^{\prime}}=J_{\mathrm{LR}}, J_{\mathrm{SR}^{\prime}}=-J_{\mathrm{SR}}, H^{\prime}=H, H=H^{\prime}$, $M^{\prime}\left(\equiv M_{1}-M_{2}\right)=M\left(\equiv M_{1}+M_{2}\right)$, and $M=M^{\prime}$. Thus, a solution to (1) and (2) is equivalently a solution to (20) with the above identifications or reinterpretation of the parameters.

It was found in Paper I that for $H^{\prime}=0, H \neq 0$ there are three qualitatively different kinds of phase behavior,

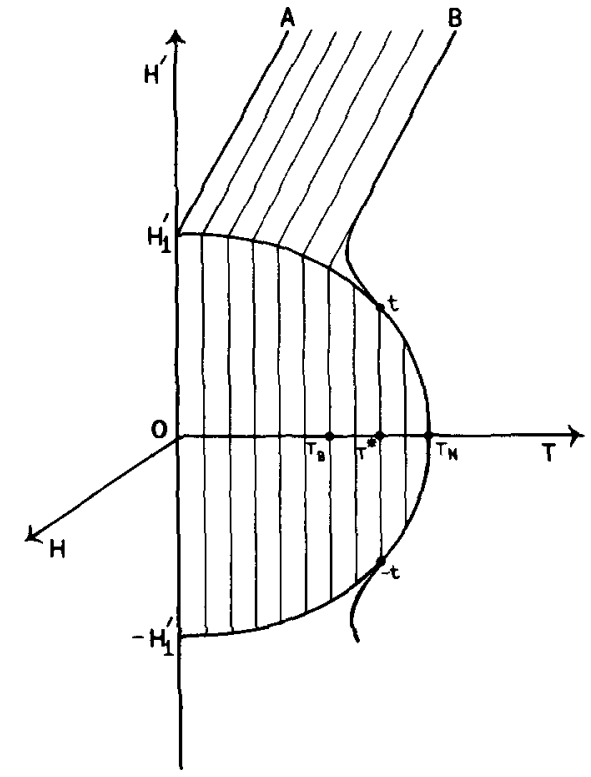

FIG. 1. The schematic $H, H^{\prime}, T$ phase diagram for $J_{\mathrm{gR}} / J_{\mathrm{LR}}>$ $-0.31714 \cdots$. The surfaces, such as $H_{1}^{\prime}, t, T_{N},-t,-H_{1}{ }^{\prime}$, are the loci of first-order or discontinuous phase transitions. The perimeters, such as $B, t$ and $t, T_{N},-t$ are lines of critical points. The surface $H_{1}^{\prime}, A ; B, t$ has three images under the symmetries $H \rightarrow-H$ and $H^{\prime} \rightarrow-H^{\prime}$ which, for convenience, are not shown. In the particular case $J_{\mathrm{SR}}=-1 / 2$ and $J_{\mathbf{L R}}=1.648721 \cdots$, one has $T_{N}=2, T^{*}=1.9421 \cdots, T_{B}=J_{\mathrm{LR}} / k$ and $H_{1}{ }^{\prime}=J_{\mathrm{LR}}+2 J_{\mathrm{SR}}$.

and this continues to be the case when $H^{\prime} \neq 0$. For fixed $J_{\mathrm{SR}}$ and $J_{\mathrm{LR}}$ and $J_{\mathrm{SR}} / J_{\mathrm{LR}}>-0.31714 \cdots$, the phase diagram in the $H, H^{\prime}, T$ variable set is shown schematically in Fig. 1. The shaded portion of the $H=0$ plane, identified in the figure by the points $H_{1}{ }^{\prime}, t, T_{N},-t,-H_{1}{ }^{\prime}$ on the perimeter of the shaded portion, is a first-order transition plane; in particular, for variables $H=0, H^{\prime}$, and $T$ lying on that shaded portion, there is a nonzero spontaneous magnetization $\pm M_{s}$. The high temperature boundary $t, T_{N},-t$ is a line of critical points; thus the differential zero field susceptibility diverges on this line, the locus of which is given by $\mathrm{Eq}$. (17). At the tricritical points $\pm t$, the coefficient $a_{4}(T)$ in Eq. (16) also vanishes. Thus, the analytic continuations of the line $t, T_{N},-t$ are metastable critical lines and are masked by the firstorder transition along the lines $H_{1}{ }^{\prime}, t$ and $-t,-H_{1}{ }^{\prime}$. The negativity of $a_{4}(T)$ while $a_{2}(T)$ is positive induces a transition in nonzero direct field $H$. This transition is represented by the surface $H_{1}{ }^{\prime}, A, B, t$, and the three other surfaces (not drawn in Fig. 1) which are related by the symmetries $H \leftrightarrow-H$ and $H^{\prime} \leftrightarrow-H^{\prime}$. This transition surface ends at $T=0$ along the firstorder line given by $H_{1}^{\prime}-H_{1}=2 J_{\mathrm{SR}}+J_{\mathrm{LR}}$. At finite temperatures one can obtain the limiting behavior of the transition surface as $H$ and $H^{\prime}$ become very large. Just as at $T=0$, purely energetic considerations yield the equation for the transition surface, $H_{1}{ }^{\prime}=H_{1}+$ $J_{\mathrm{LR}}+2 J_{\mathrm{SR}}$. However, the transition surface for large $H$ and $H^{\prime}$ only extends up to the temperature $k T_{c}=J_{\mathrm{LR}}$ 


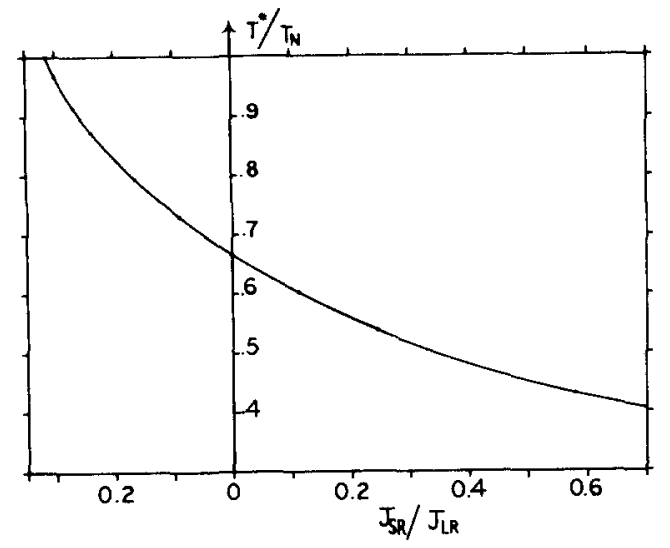

FIG. 2. The variation of $T^{*} / T_{N}$ with $J_{\mathrm{SR}} / J_{\mathrm{LR}}$.

and the critical magnetization is given by $M_{c}=1 / 2$. These relations were discovered empirically, but they can be shown to be consistent with the sixth-order stability. equation $a_{2}(T)=0$ in nonzero fields when $L$ and $L^{\prime}$ are much greater than one.

The tricritical points $\pm t$ occur at the ends of the triple lines $H_{1}{ }^{\prime}, t$ and $-H_{1}{ }^{\prime},-t$, each point of which is a proper triple point. The relative locations of the tricritical points to the Néel point can be expressed by the ratio $T^{*} / T_{N}$, following the notation used to describe metamagnets. ${ }^{9}$ In much work on metamagnets, the basic variable which controls $T^{*} / T_{N}$ is the ratio $\Gamma / A$ in Eq. (18). In this work the basic variable is $J_{\mathrm{SR}} / J_{\mathrm{LR}}$. In Fig. 2 the variation of $T^{*} / T_{N}$ is plotted versus $J_{\mathrm{SR}} / J_{\mathrm{LR}}$. The case $J_{\mathrm{SR}} / J_{\mathrm{LR}}=0$ was originally solved by Sauer and Temperley. ${ }^{13}$ For $J_{\mathrm{SR}} / J_{\mathrm{LR}}>0$, i.e., when the long-range and short-range interactions cooperate rather than compete, the tricritical point recedes from the Néel point towards $T=0$. As $J_{\mathrm{SR}} / J_{\mathrm{L} \text {. }}$ goes negative, i.e., when the long-range and shortrange interactions compete, the tricritical point approaches the Néel point. For the ratio $J_{\mathrm{SR}} / J_{\mathrm{LR}}=$ $-0.31714 \cdots$, which in Paper I was called the confluent point, the tricritical points merge with the Néel point. In an $H, H^{\prime}, T$ phase diagram this confluent point is technically a tetracritical point in the sense that it is the meeting point of four critical lines. For $-1 / 2<J_{\mathrm{SR}} / J_{\mathrm{LR}}<-0.31714 \cdots$ the tricritical points disappear altogether, resulting in the phase diagram shown schematically in Fig. 3. In this case the transition in zero fields $H^{\prime}=0$ and $H=0$ at $T_{1}$ is first order and is technically a triple point on a triple line where three first-order surfaces meet. For $J_{\mathrm{SR}} / J_{\mathrm{IR}}<-1 / 2$ the ground state in zero field becomes antiferromagnetic and the transition in zero field, i.e., the surface $H_{1}$, $T_{1},-H_{1}{ }^{\prime}$ disappears, leaving two disjoint phase surfaces in a field $H \neq 0$.

As was discussed in Paper I, the critical exponents at ordinary critical points on these phase diagrams have the usual classical values $\beta=1 / 2, \gamma=1=\gamma^{\prime}$, $\delta=3$, and $\alpha=0=\alpha^{\prime}$ (finite). ${ }^{11}$ However, in the present paper, at the confluent point and also at the tricritical points the exponents take on the exceptional values $\beta=1 / 4, \gamma=1=\gamma^{\prime}, \delta=5$, and $\alpha=0, \alpha^{\prime}=1 / 2 .{ }^{14}$ Although these specific values of the tricritical exponents are expected to apply only to classical models, topological considerations suggest that tricritical exponents may be different from the usual critical exponents even for more realistic models and for real systems.

Since more realistic models with tricritical points have not been solved, it may be of some value to discuss the question of the observability of the tricritical exponents using this model as a guide. The following computed examples are for the case $J_{\mathrm{SR}}=-1 / 2$ and $J_{\mathrm{LR}}=1.64872 \cdots$ for which the critical point in zero fields ( $T_{N}$ in metamagnetic language) is $T_{N}=2.0$ and $T^{*}=1.9421864 \cdots$. In Fig. 4 a double log plot of the spontaneous magnetization versus $T_{c}\left(H^{\prime}\right)-T$ is shown, where $T_{c}\left(H^{\prime}\right)$ is the critical temperature in a field $H^{\prime}$. In metamagnetic language, the spontaneous magnetization is the sublattice magnetization as measured by neutron diffraction or nuclear magnetic resonance, $H^{\prime}$ is the direct field, and $T_{c}\left(H^{\prime}\right)$ is the Néel temperature in a field. For $T_{c}\left(H_{t}^{\prime}\right)=T^{*}$, i.e., right at the tricritical field $H_{t}^{\prime}$, the curve in Fig. 4 has a slope of about 0.28 in the $0.1-0.01$ decade and a slope very close to 0.25 in the decades closer to $T^{*}$. For $T_{e}(0)=T_{N}$ the curve in Fig. 4 does not attain its asymptotic value of $1 / 2$ until $\left|T-T_{N}\right| \simeq 0.001$, but it is easy to see, even on the basis of the values $\left|T-T_{N}\right| \geq 0.01$, that the two curves are likely to have different exponents. It is much harder to conclude

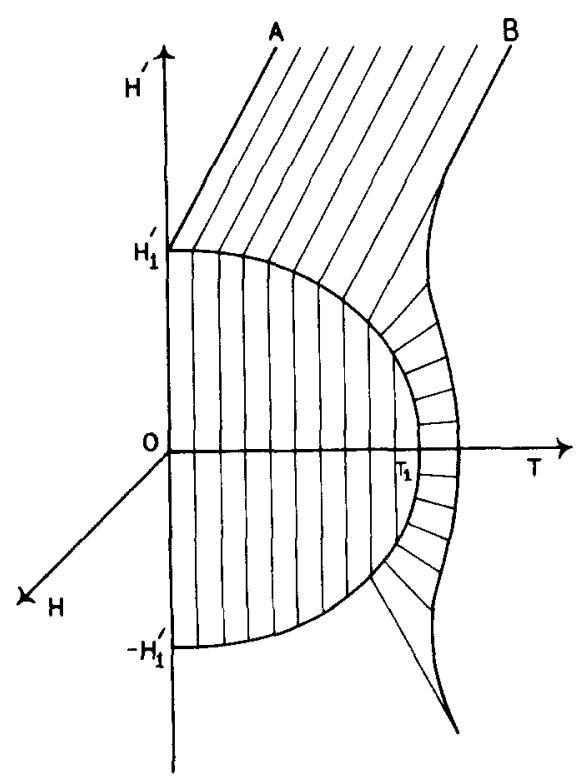

FIg. 3. The schematic $H, H^{\prime}, T$ phase diagram for $-1 / 2<$ $J_{\mathrm{SR}} / J_{\mathrm{LR}}<-0.31714 \cdots$. In the particular case $J_{\mathrm{SR}}=-1 / 2$ and $J_{\mathrm{LR}}=1.434923 \cdots$, one has $T_{N}=1.5, T_{B}=J_{\mathrm{LR}} / k$. 


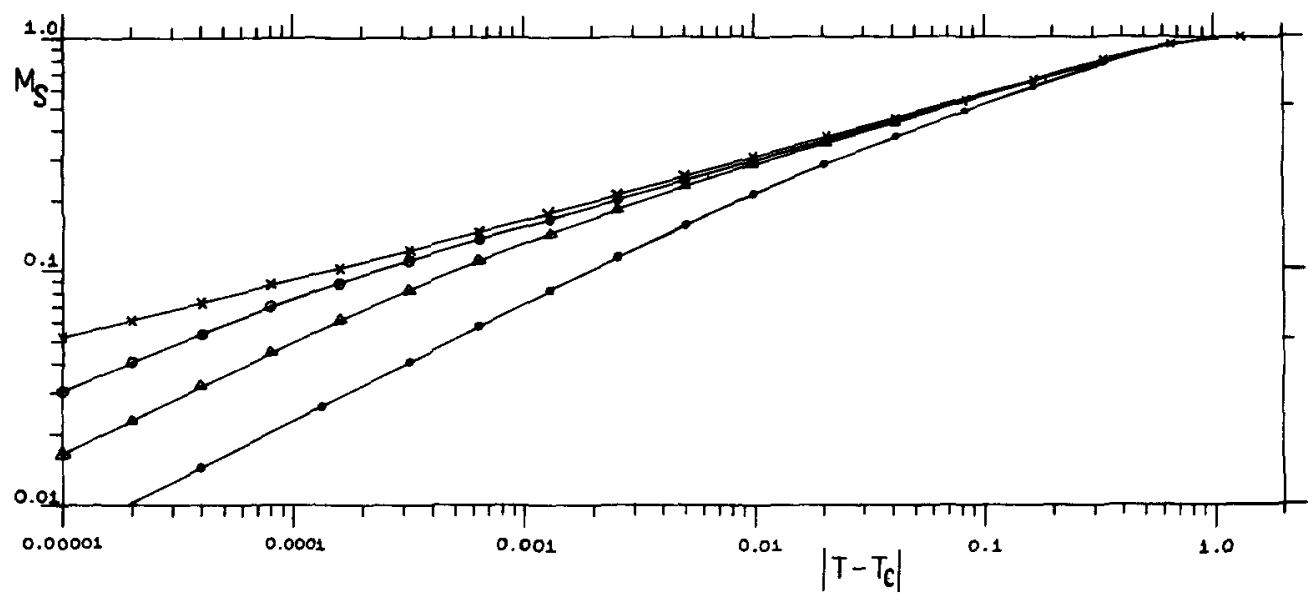

FIg. 4. $\log M_{S}$ versus $\log \left[T_{c}\left(H^{\prime}\right)-T\right]$, where $T_{c}\left(H^{\prime}\right)$ is the critical temperature in a field $H^{\prime}$ for $H=0$, for the case $J_{\mathrm{SR}}=-1 / 2$ and $J_{\mathrm{LR}}=1.64872 \cdots$. From top to bottom the critical temperatures are $T_{c}=T^{*}, T^{*}+2 \frac{1}{2} \mathrm{mdeg}, T^{*}+10 \mathrm{mdeg}$, and $T_{N} \simeq T^{*}+58 \mathrm{mdeg}$.

that $\beta=1 / 2$ for the curves for $T_{c}\left(H^{\prime}\right)$ closer to $T^{*}$, i.e., for $T_{c}\left(H^{\prime}\right)=T^{*}+2 \frac{1}{2} \mathrm{mdeg}$ and $T^{*}+10 \mathrm{mdeg}$, unless one can measure more decades in $\left|T-T_{c}\left(H^{\prime}\right)\right|$. From the point of view of studying tricritical points, however, this should be considered an advantage because there will probably be some experimental error in determining $T^{*}$. For example, if there is a $2 \frac{1}{2}$-mdeg error in determining $T^{*}$, and the measurements only go in to $\left|T-T_{\mathrm{c}}\right|=0.001$, one will still obtain a fairly good estimate $(0.29)$ of the tricritical exponent $\beta$. Thus, it is to be hoped that one will be able experimentally to distinguish the tricritical exponent $\beta$ from the usual critical exponent $\beta$, even though there may be some difficulty in locating the tricritical point very precisely.

In Fig. 5 is shown a similar double log plot of the specific heat versus $T_{c}\left(H^{\prime}\right)-T$, where the $T_{c}\left(H^{\prime}\right)$ are the same as in Fig. 1, namely, $T_{c}\left(H^{\prime}\right)=T^{*}, T^{*}+2 \frac{1}{2}$ mdeg, $T^{*}+10 \mathrm{mdeg}$, and $T_{N}$. Also for comparison the curve for the more customary $J_{\mathrm{SR}}=0, J_{\mathrm{LR}}=1$ case is shown. If measurements go to within $0.0001^{\circ} \mathrm{K}$ of $T_{c}$ and even if $T^{*}$ is overestimated by $2 \frac{1}{2} \mathrm{mdeg}$, one would obtain an estimate for $\alpha^{\prime}$ of 0.45 . We do not present similar graphs for other critical exponents because they either do not change in this model, as is the case for the susceptibility exponent, or they

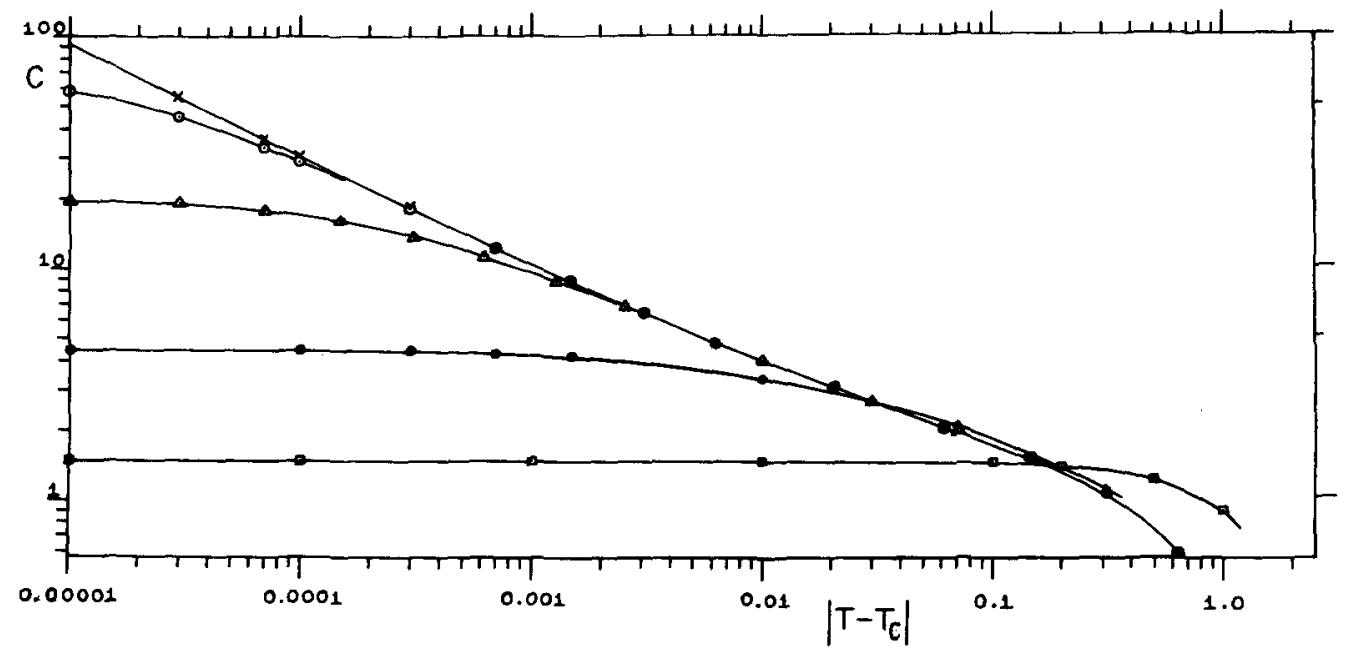

Fig. 5. $\log C_{B-0, B}$, versus $\log \left[T_{c}\left(B^{\prime}\right)-T\right]$ for the same cases as in Fig. 4, except that the lowest curve is obtained from $J_{\mathrm{SR}}=0, T_{c}=T_{N}$. 
are very difficult to observe experimentally, such as the metamagentic $M^{\prime}$ versus $H^{\prime}$ critical isotherm.

* Work supported in part by National Science Foundation Grant GP-21093.

$\dagger$ Alfred P. Sloan Research Fellow.

1 J. F. Nagle, Phys. Rev. A 2, 2124 (1970), Paper I.

2 This fact seems to be generally well known. It is formalized to varying degrees in Refs. $3-5$.

${ }^{3} \mathrm{~J}$. W. Stout and R. C. Chisholm, J. Chem. Phys. 36, 979 (1962).

${ }^{4}$ G. A. Baker, Jr., Phys. Rev. 130, 1406 (1963).

${ }^{5}$ M. Suzuki, J. Phys. Soc. Japan 21, 2140 (1966).

${ }^{6}$ S. Katsura, Phys. Rev. 127, 1508 (1962). Very long range interactions as well as a staggered field were added by Suzuki, Ref. 5 , but he overlooked the in teresting phase behavior for competing interactions; this topic will be discussed by the authors in a separate paper.

7 P. C. Hemmer and G. Stell, Phys. Rev. Letters 24, 1285 (1970).

${ }^{8}$ R. B. Griffiths, Phys. Rev. Letters 24, 715 (1970).

O I. S. Jacobs and P. E. Lawrence, Phys. Rev. 164, 866 (1967).
${ }^{10} \mathrm{~V}$. A. Schmidt and S. A. Friedberg, Phys. Rev. B 1, 2250 (1970) . B. E. Keen, D. Landau, B. Schneider, and W. P. Wolf, J. Appl. Phys. 37, 1120 (1966).

${ }_{11}$ M. E. Fisher, Rept. Progr. Phys. 30, 615 (1967).

12 See, for example, J. Rowlinson, Liquids and Binary Mixtures (Butterworths, London, 1969). The double tangent construction, which is equivalent to the Maxwell construction, is subject to a certain amount of ambiguity when only the equation of state is known, R. B. Griffiths, Phys. Rev. 158, 176 (1967), but this is no problem here because we start from the free energy. The very existence of the van der Waals loops can also be criticized as not being physical (Fisher, Ref. 11). However, if instead of using the equivalent neighbor potential, the very long range Kac-Baker $\gamma e^{-\gamma \tau}$ potential is used in the appropriate limits, then it follows from the work of G. A. Baker, Jr., Ref. 4, and J. Lebowitz and $O$. Penrose, J. Math. Phys. 7, 98 (1966) that the convex envelope of $G_{1}$ versus $M$ is obtained directly.

${ }_{13} \mathrm{~J}$. A. Sauer and H. N. V. Temperley, Proc. Roy. Soc. (London) A176, 203 (1940).

${ }_{14}$ This set of exponent values was discussed, independently of any model, by Rowlinson, Ref. 12. For a discussion of their relevance to scaling-homogeneity theory see Ref. 7.

\title{
Infrared Spectra of Divalent Metal Monothioacetylacetonates
}

\author{
Olavi Simann and James Fresco \\ Chemistry Department, McGill University, Montreal, Canada
}

(Received 22 December 1969)

\begin{abstract}
The infrared spectra of copper (II), nickel (II), zinc (II), palladium (II), cadmium (II), and lead (II) complexes of monothioacetylacetone have been recorded from $1700-300 \mathrm{~cm}^{-1}$. Assignments for in-plane vibrations were obtained by normal coordinate analyses. The carbon-sulfur stretching vibration was located near $710 \mathrm{~cm}^{-1}$. In every case metal-oxygen stretching force constants were larger than metalsulfur constants and carbon-sulfur constants were less than carbon-oxygen constants. Systematic trends which appeared among both metal-sulfur and metal-oxygen stretching force constants were correlated with sequences observed among the carbon-donor atom force constants.
\end{abstract}

\section{INTRODUCTION}

Although the preparation of sulfur analogs of $\beta$ diketones and their chelates ${ }^{1}$ has been the focus of a number of recent investigations, detailed information on the properties and bonding in these compounds is meager. Recently, the type of normal coordinate analysis developed by Nakamoto for studies of the acetylacetonates $^{2}$ was applied to several newly synthesized chelates of dithioacetylacetone. ${ }^{3}$ The study showed that while metal-sulfur stretching force constants increase in a predictable way, their values were significantly less than the metal-oxygen force constants of the corresponding acetylacetonates.

Since the properties of the chelates of monothioacetylacetone differ considerably from those of acetylacetone and dithioacetylacetone, a vibrational analysis of the monothioacetylacetonates should further contribute to understanding the effect of sulfur-for-oxygen replacement in the chelates of $\beta$-diketones. In contrast to acetylacetonates and dithioacetylacetonates, monothioacetylacetonates belong to a point group of lower symmetry. In previous cases where the infrared spectra for the latter compounds were reported, a absorption bands were assigned on a strictly empirical basis. The carbon-sulfur stretching mode which was tentatively assigned to the band near $1220 \mathrm{~cm}^{-1}$ has now been shown to appear close to $710 \mathrm{~cm}^{-1}$. The values of stretching force constants, $K(\mathrm{C} \cdots \mathrm{S})$, indicate predominantly single-bonded carbon-sulfur in the metal chelates.

In the present study the infrared absorption spectra of bis(monothioacetylacetonato) complexes of nickel(II), copper(II), zinc(II), palladium(II), cadmium(II), and lead(II) have been recorded and theoretical assignment of in-plane chelate ring vibrations has been made.

\section{EXPERIMENTAL SECTION}

\section{Preparation of Compounds}

All the complexes except that of copper(II) were prepared by adding $0.01 \mathrm{~mol}$ metal nitrate dissolved in 